\title{
THE ANALYST.
}

\section{PROCEEDINGS OF THE SOCIETY OF PUBLIC ANALYSTS AND OTHER ANALYTICAL CHEMISTS.}

\section{THE QUANTITATIVE SEPARATION OF LEAD AND BISMUTH.*}

\author{
By J. C. GALLETLY and G. G. HENDERSON, M.A., D.Sc., F.I.C.
}

IT is known that many metals can be estimated with accuracy in the form of their phosphates, and the method appears to be particularly suitable for the estimation of bismuth, which is precipitated quantitatively from solutions of its chloride or nitrate by addition of sodium phosphate (Staehler and Scharfenberg, Ber., 1905, 38, 3862 ; Salkowski, idem., 3943). Moreover, since bismuth phosphate is practically insoluble in dilute phosphoric acid, whilst the phosphates of a number of other metals are dissolved more or less readily by that reagent, the separation of those metals from bismuth can be effected without trouble. Lead phosphate, however, is only sparingly soluble in phosphoric acid, and therefore the attempts which have been made hitherto to separate the phosphates of lead and bismuth have given unsatisfactory results. Staehler and Scharfenberg have suggested that the separation might be effected by precipitating the phosphates together, and then removing the lead phosphate by boiling the precipitate with 1 per cent. nitric acid. Following up this suggestion, we have made a number of experiments on the separation of the phosphates of lead and bismuth, and have also tested some of the other methods which have been proposed for the estimation of bismuth in presence of lead.

Preliminary experiments, of which the details are omitted, confirmed the statements that the precipitation of both lead and bismuth phosphates is quantitative, and that bismuth phosphate is insoluble, and lead phosphate only sparingly soluble, in dilute phosphoric acid. It was also found that lead phosphate dissolves slowly but completely, whilst bismuth phosphate is hardly attacked, when heated with 1 per cent. nitric acid, and that the former salt is readily dissolved by 3.5 per cent. nitric acid in the cold, but the latter only to a small extent.

In order to ascertain if a separation of the metals could be effected by precipitating bismuth phosphate in presence of sufficient nitric acid to retain lead phosphate in solution, a series of experiments was carried out with solutions containing from

\footnotetext{
* This work was carried out in accordance with the terms of the "Analytical Investigation Scheme."
} 
0.5 to 5 per cent. of the acid. It was found that with the weaker solutions the results for bismuth were too high, and with the stronger solutions too low, but on precipitating the bismuth from a solution containing 2.5 per cent. of nitric acid, and subsequently digesting the precipitate with a little more acid of the same concentration, the following figures were obtained:

$\begin{array}{cccc}\mathrm{Pb} \text { Taken. } & \text { Bi Taken. } & \text { Bi Found. } & \text { Difference } \\ \cdot 24549 & \cdot 24163 & \cdot 24286 & \cdot 00123+\end{array}$

In view of this result a trial was given to the following method: The solution of the nitrates of the metals was mixed with so much nitric acid that, after addition of the necessary quantity of sodium phosphate, the liquid still contained about 2.5 per cent. of nitric acid. The hot solution of sodium phosphate was added to the boiling solution of the nitrates, and the mixture was boiled for five to ten minutes, allowed to settle on the water-bath, and decanted through a Gooch crucible. The precipitate was digested on the water-bath for about ten minutes with 10 to 15 c.c. of 2.5 per cent. nitric acid, transferred to the crucible, washed with water containing a little nitric acid and a trace of ammonium nitrate, ignited, and weighed as $\mathrm{BiPO}_{4}$. The results were disappointing, for whilst in some of the experiments fairly close agreement was reached, there was on the whole considerable variation in the percentage of bismuth found. The following results may be taken as typical of a large number:

\begin{tabular}{|c|c|c|c|}
\hline Pb Taken. & Bi Taken. & Bi Found. & Difference \\
\hline$\cdot 23684$ & $\cdot 25721$ & $\cdot 25866$ & $\cdot 00145+$ \\
\hline$\cdot 23684$ & $\cdot 10288$ & $\cdot 10187$ & $\cdot 00101-$ \\
\hline .23684 & $\cdot 10288$ & $\cdot 10351$ & $.00063+$ \\
\hline$\cdot 23684$ & .05144 & $\cdot 04819$ & $\cdot 00325$ \\
\hline •23684 & $\cdot 05144$ & $\cdot 04861$ & $\cdot 00283-$ \\
\hline .94736 & $\cdot 10288$ & $\cdot 10311$ & $\cdot 00023+$ \\
\hline$\cdot 94736$ & $\cdot 10288$ & $\cdot 10640$ & $\cdot 00352+$ \\
\hline $2 \cdot 36840$ & •10288 & •10901 & \\
\hline
\end{tabular}

In a number of other experiments we tried the effect of varying the quantity of acid with which the precipitate was digested, the duration of the process, and, when the proportion of lead was large, the number of digestions; finally, we came to the conclusion that, although it is possible to get fairly accurate results, the process is, on the whole, too troublesome for ordinary use on account of the necessity for so carefully regulating the percentage of nitric acid in the solution.

A simpler process was then adopted. A boiling solution of sodium phosphate, in sufficient quantity to precipitate the lead and bismuth completely, was added to the boiling solution of the nitrates, and the mixture was boiled for five to ten minutes and left to settle on the water-bath. The liquid was decanted through a Gooch crucible, and the precipitate was washed by decantation. The subsequent treatment of the precipitate was varied in several ways : in some experiments 1 per cent. nitric acid, in others 2.5 per cent. nitric acid was used; in some cases the precipitate was boiled with the acid, in others it was digested on the water-bath with constant stirring; in some experiments the precipitate was treated once with the acid, in others the boiling or digestion was repeated for a second or a third time, the 
duration of the treatment being also varied in different sets of determinations. The results were again disappointing, for although satisfactory figures were obtained in some cases, the variations in the percentage of bismuth found under similar conditions of experiment were so great that the process was abandoned.

After some trials we also abandoned the following methods :

1. Precipitation of lead by ammonium persulphate, the bismuth being stated to remain in solution (Dittrich and Reise, Ber., 1905, 38, 1829).

2. Electrolytic separation of bismuth and lead according to Balachowsky (Comptes Rendus, 1900, [3], 131, 179).

3. Electrolytic separation according to Hollard and Bertiaux (Comptes Rendus, 1904, [3], 139, 366).

Finally, a number of estimations of bismuth in presence of lead were carried out by the method proposed by Clark (J. Soc. Chem. Ind., 1900, 19, 26). A solution of the chlorides of lead and bismuth containing a spiral of steel wire (1 to 2 grms.) was heated until most of the iron was dissolved, and the precipitated bismuth and the remainder of the iron were collected, washed with boiling water until free from lead, and dissolved in hydrochloric acid with the aid of potassium chlorate. After diluting the solution the bismuth was precipitated as sulphide, the precipitate washed with a solution of hydrogen sulphide until free from iron, dissolved in nitric acid, and after filtration precipitated with ammonium carbonate. The precipitate was washed, ignited, and weighed as $\mathrm{Bi}_{2} \mathrm{O}_{3}$. The results obained, which are given in the following table, were much more satisfactory than those given by any other of the processes tried :

\begin{tabular}{|c|c|c|c|}
\hline Pb Taken. & Bi Taken. & Bi Found. & Difference \\
\hline$\cdot 29216$ & $\cdot 29216$ & $\cdot 29207$ & .00009 \\
\hline$\cdot 29216$ & $\cdot 29216$ & $\cdot 29316$ & .00100 \\
\hline .29216 & $\cdot 11686$ & $\cdot 11887$ & .00201 \\
\hline .29216 & $\cdot 11686$ & $\cdot 11601$ & .00075 \\
\hline $1 \cdot 16860$ & $\cdot 11686$ & $\cdot 11421$ & .00265 \\
\hline $1 \cdot 16860$ & $\cdot 11686$ & $\cdot 11833$ & .00147 \\
\hline $2 \cdot 92150$ & $\cdot 11686$ & $\cdot 11744$ & .00078 \\
\hline $2 \cdot 92150$ & $\cdot 11686$ & $\cdot 11726$ & $\cdot 00040$ \\
\hline $5 \cdot 84300$ & $\cdot 11686$ & $\cdot 11565$ & $\cdot 00121$ \\
\hline $5 \cdot 84300$ & $\cdot 11686$ & $\cdot 11780$ & $\cdot 00094$ \\
\hline
\end{tabular}

In conclusion, our attempts to separate lead and bismuth quantitatively by the phosphate method were unsuccessful, as was also the case with some of the other methods examined. On the other hand, we consider that Clark's process for the estimation of bismuth in presence of lead is not only easily carried out, but that it is also capable of yielding accurate results. The process, however, can be improved by precipitating the bismuth as phosphate-a method which we have found to give excellent results - instead of with ammonium carbonate, as originally described.

Department of Chemistix, Techyical College, Glasgow. 